\title{
SINGULAR INTEGRAL OPERATORS WITH ROUGH CONVOLUTION KERNELS
}

\author{
ANDREAS SEEGER
}

\section{INTRODUCTION}

The purpose of this paper is to investigate the behavior on $L^{1}\left(\mathbb{R}^{d}\right), d \geq 2$, of a class of singular convolution operators which are not within the scope of the standard Calderón-Zygmund theory.

An important special case occurs if the convolution kernel $K$ is homogeneous of degree $-d$. Suppose that $\Omega \in L^{1}\left(S^{d-1}\right)$ and

$$
\int_{S^{d-1}} \Omega(\theta) d \theta=0
$$

here $d \theta$ denotes surface measure on the sphere. Then it is easy to see that for $f \in C_{0}^{\infty}\left(\mathbb{R}^{d}\right)$ the principal value integral

$$
T_{\Omega} f(x)=\text { p.v. } \int \Omega(y /|y|)|y|^{-d} f(x-y) d y
$$

exists for all $x \in \mathbb{R}^{d}$. Calderón and Zygmund [1] used the method of rotations to show that if $\Omega \in L^{1}\left(S^{d-1}\right)$ and if the even part of $\Omega$ belongs to the class $L \log L\left(S^{d-1}\right)$, then $T$ extends to a bounded operator on $L^{p}\left(\mathbb{R}^{d}\right), 1<p<\infty$.

Proposition. Suppose that $\Omega \in L \log L\left(S^{d-1}\right)$ and suppose that the cancellation property (1.1) holds. Then $T_{\Omega}$ extends to an operator of weak type $(1,1)$.

In two dimensions this result was previously obtained by Christ and Rubio de Francia [3], and, under a slightly stronger hypothesis, by Hofmann [6]. In [2], [3] a weak type $(1,1)$ inequality was also proved for the less singular maximal operator

$$
M_{\Omega} f(x)=\sup _{r>0} r^{-d} \int_{|y| \leq r}|\Omega(y /|y|) f(x-y)| d y,
$$

in all dimensions, again under the assumption $\Omega \in L \log L$. It is conceivable that a variant of the arguments in [3] for the maximal operator could also work for the singular integral operator; in fact, in unpublished work, the authors of [3] obtained a weak type $(1,1)$ inequality in dimension $d \leq 7$. However their arguments - if applied to the singular integral operator - lead to substantial technical difficulties and no

Received by the editors August 5, 1994.

1991 Mathematics Subject Classification. Primary 42B20; Secondary 42B25.

Research supported in part by a grant from the National Science Foundation. 
proof has been known for the higher-dimensional cases. In this paper we develop a different and conceptually simpler method, based on a microlocal decomposition of the kernel (cf. (2.2) below). Incidentally this method also gives a new proof of the weak type bounds for $M_{\Omega}$.

The proposition is a special case of a more general theorem concerning translation invariant operators $T$ with rough convolution kernels $K \in \mathcal{S}^{\prime}$. We assume that $K$ is locally integrable away from the origin, so that

$$
\langle T f, g\rangle=\iint g(x) f(y) K(x-y) d y d x
$$

for all $f, g \in C_{c}^{\infty}\left(\mathbb{R}^{d}\right)$ with disjoint supports. Clearly $T$ extends to a bounded operator on $L^{2}\left(\mathbb{R}^{d}\right)$ if and only if the Fourier transform $\widehat{K}$ belongs to $L^{\infty}\left(\mathbb{R}^{d}\right)$. Introducing polar coordinates $x=r \theta, r>0, \theta \in S^{d-1}$, we shall assume a weak regularity condition for $r \mapsto K(r \theta)$. However only size conditions will be imposed in the $\theta$ variable.

In order to formulate our assumptions let

$$
V_{R}(\theta)=\int_{R}^{2 R}|K(r \theta)| r^{d-1} d r
$$

and

$$
V(\theta)=\sup _{R>0} V_{R}(\theta)
$$

Moreover, for $a \geq 2$ let

$$
\eta(a)=\sup _{R \geq a s} \int_{S^{d-1}} \int_{R}^{2 R}|K((r-s) \theta)-K(r \theta)| r^{d-1} d r d \theta .
$$

We shall always assume the Dini-condition

$$
\int_{2}^{\infty} \eta(a) \frac{d a}{a}<\infty
$$

Theorem. Suppose that $T$ is as in (1.3) and that $\widehat{K} \in L^{\infty}\left(\mathbb{R}^{d}\right)$. Suppose that (1.7) holds and that $V \in L \log L\left(S^{d-1}\right)$. Then $T$ is bounded in $L^{p}, 1<p<\infty$; moreover, $T$ is of weak type $(1,1)$.

Note that for the operators in (1.2) we have $\eta(a)=O\left(a^{-1}\right)$ and $V=c|\Omega|$. Therefore the Proposition follows from the Theorem.

Remarks. (i) It may be more natural to impose an integrability condition on $V_{R}$, uniformly in $R$, rather than on the maximal quantity $V$. Indeed the hypothesis $V \in L \log L$ can be replaced by

$$
\sup _{R} \int_{S^{d-1}} V_{R}(\theta)\left(1+\Delta\left(V_{R}(\theta) /\left\|V_{R}\right\|_{1}\right)\right) d \theta<\infty
$$


for some nondecreasing function $\Delta:[1, \infty) \rightarrow(0, \infty)$ satisfying

$$
\int_{1}^{\infty} \frac{d a}{a \Delta(a)}<\infty
$$

Typical choices for $\Delta$ are

$$
\begin{aligned}
& \Delta(t)=\log ^{1+\varepsilon}(2+t), \text { or } \\
& \Delta(t)=\log (2+t) \log \left(2+\log ^{1+\varepsilon}(2+t)\right), \text { etc. }
\end{aligned}
$$

(ii) Without the assumption $\Omega \in L \log L\left(S^{d-1}\right)$ even the $L^{2}$ boundedness of $T_{\Omega}$ may fail. This was pointed out by Calderón and Zygmund [1]. However if $\Omega \in L^{1}\left(S^{d-1}\right)$ is odd, then $T_{\Omega}$ is bounded on $L^{p}, 1<p<\infty$ (see [1]). Presently it is not known whether a weak type $(1,1)$ inequality holds in this case.

In $\S 2$ we shall give the main estimates needed to prove the Theorem. The formal proof is contained in $\S 3$.

The following notation is used: For a set $E \subset \mathbb{R}^{d}$ we denote the Lebesgue measure of $E$ by $|E|$. For a set $A \in S^{d-1}$ we also write $|A|=\int_{A} d \theta$. The Fourier transform of $f$ is denoted by $\widehat{f}$, the inverse Fourier transform of $f$ is denoted by $\mathcal{F}^{-1}[f]$. Given two quantities $a$ and $b$ we write $a \lesssim b$ or $b \gtrsim a$ if there is a positive constant $C$, depending only on the dimension, such that $a \leq C b$. We write $a \approx b$ if $a \lesssim b$ and $a \gtrsim b$.

\section{Main estimates}

Let $\left\{H_{j}\right\}$ be a family of functions with

$$
\operatorname{supp} H_{j} \subset\left\{x: 2^{j-2} \leq|x| \leq 2^{j+2}\right\} .
$$

We assume that the $H_{j}$ are differentiable in the radial variable and that the estimates

$$
\sup _{0 \leq l \leq N} \sup _{j} r^{d+l}\left|\left(\frac{\partial}{\partial r}\right)^{l} H_{j}(r \theta)\right| \leq \mathfrak{M}_{N}
$$

hold uniformly in $\theta$ and $r$. Convolution kernels of this type come up in a dyadic decomposition of the kernel of the operator defined in (1.2), if $\Omega \in L^{\infty}\left(S^{d-1}\right)$.

We shall be interested in estimates for $H_{j} * \sum_{Q} b_{Q}$ where each $b_{Q}$ is a building block in a Calderón-Zygmund decomposition, supported in a cube $Q$, and where the sidelength $2^{L(Q)}$ of $Q$ is small compared to the diameter of supp $H_{j}$; say by a factor of $\approx 2^{-s}$.

For $s>3$ let $\mathfrak{E}^{s}=\left\{e_{\nu}^{s}\right\}$ be a collection of unit vectors with mutual distance $>2^{-s-10} d^{-1}$ such that for each $\theta \in S^{d-1}$ there is an $e_{\nu}^{s}$ with $\left|\theta-e_{\nu}^{s}\right| \leq 2^{-s-1}$. It is easy to see that we may construct disjoint measurable sets $E_{\nu}^{s} \subset S^{\bar{d}-1}$ with $e_{\nu}^{s} \in E_{\nu}^{s}, \operatorname{diam}\left(E_{\nu}^{s}\right) \leq 2^{-s}$ and $\bigcup_{\nu} E_{\nu}^{s}=S^{d-1}$. Then clearly

$$
\operatorname{card}\left(\mathfrak{E}^{s}\right) \approx 2^{s(d-1)} .
$$

Let

$$
H_{j \nu}^{s}(x)=H_{j}(x) \chi_{E_{\nu}^{s}}(x /|x|) .
$$


A further decomposition will be based on the observation that the Fourier transform $\widehat{H_{j \nu}^{s}}$ is concentrated near the hyperplane perpendicular to $e_{\nu}^{s}$.

Fix a parameter $\kappa$, such that $0<\kappa<1$. Let $\psi \in C_{0}^{\infty}(\mathbb{R})$ be supported in $[-4,4]$ such that $\psi(t)=1$ for $t \in[-2,2]$. Define $P_{\nu}^{s}$ by

$$
\widehat{P_{\nu}^{s}}(\xi)=\psi\left(2^{s(1-\kappa)}\left\langle\xi, e_{\nu}^{s}\right\rangle /|\xi|\right) .
$$

Our basic splitting is

$$
H_{j}=\Gamma_{j}^{s}+\left(H_{j}-\Gamma_{j}^{s}\right)
$$

where

$$
\Gamma_{j}^{s}=\sum_{\nu} P_{\nu}^{s} * H_{j \nu}^{s} .
$$

Lemma 2.1. Let $\mathfrak{Q}$ be a collection of cubes $Q$ with disjoint interiors. Define $L(Q)=m$ if $2^{m-1}<\operatorname{sidelength}(Q) \leq 2^{m}$ and let $\mathfrak{Q}_{m}=\{Q \in \mathfrak{Q}: L(Q)=m\}$. For each $Q$ let $f_{Q}$ be an integrable function supported in $Q$ satisfying

$$
\int\left|f_{Q}(x)\right| d x \leq \alpha|Q|
$$

Let $F_{m}=\sum_{Q \in \mathfrak{Q}_{m}} f_{Q}$. Then for $s>3$

$$
\left\|\sum_{j} \Gamma_{j}^{s} * F_{j-s}\right\|_{2}^{2} \leq C\left[\mathfrak{M}_{0}\right]^{2} 2^{-s(1-\kappa)} \alpha \sum_{Q}\left\|f_{Q}\right\|_{1} .
$$

In our application of Lemma 2.1 the functions $f_{Q}$ will be the basic building blocks which arise in a Calderón-Zygmund decomposition at height $c \alpha$. Note however that for this part no cancellation condition for $f_{Q}$ is assumed.

Lemma 2.2. Let $Q$ be a cube of sidelength $2^{j-s}$ and let $b_{Q}$ be integrable and supported in $Q ;$ moreover, suppose that $\int b_{Q}=0$. Then for $N \geq d+1$ and $0 \leq \varepsilon \leq 1$

$$
\left\|\left(H_{j}-\Gamma_{j}^{s}\right) * b_{Q}\right\|_{1} \leq C_{N}\left[\mathfrak{M}_{0} 2^{-s \varepsilon}+\mathfrak{M}_{N} 2^{s(d+(\varepsilon-\kappa) N)}\right]\left\|b_{Q}\right\|_{1}
$$

where $C_{N}$ does not depend on $j$ or $Q$.

It is important to keep track of how the estimates depend on $\mathfrak{M}_{N}$ since we shall apply the lemmas in a situation where this norm is large and depends on $s$ itself. The bounds in Lemma 2.2 are not best possible, but this is irrelevant for our purpose.

Proof of Lemma 2.1. We use an orthogonality argument based on the following observation. Given $s>3$, each $\xi \neq 0$ is contained in at most $C 2^{s(d-2+\kappa)}$ of the sets supp $\widehat{P_{\nu}^{s}}$ where $C$ only depends on $d$. In fact by homogeneity it suffices to check this for $\xi \in S^{d-1}$. If $\xi \in \operatorname{supp} \widehat{P_{\nu}^{s}} \cap S^{d-1}$ and $\xi^{\perp}$ is the hyperplane perpendicular to $\xi$, then

$$
\operatorname{dist}\left(e_{\nu}^{s}, \xi^{\perp}\right) \leq c 2^{-s(1-\kappa)} .
$$


Since the mutual distance of the $e_{\nu}^{s}$ is bounded below by $c^{\prime} 2^{-s}$ there are at most $c^{\prime \prime} 2^{s(d-2+\kappa)}$ of the $e_{\nu}^{s}$ satisfying (2.3). This implies the observation.

We apply Plancherel's theorem, the Cauchy-Schwarz inequality and then Plancherel's theorem again to obtain

$$
\begin{aligned}
\left\|\sum_{j} \Gamma_{j}^{s} * F_{j-s}\right\|_{2}^{2} & =(2 \pi)^{d / 2}\left\|\sum_{\nu} \widehat{P_{\nu}^{s}} \sum_{j} \widehat{H_{j \nu}^{s}} \widehat{F_{j-s}}\right\|_{2}^{2} \\
& \leq C 2^{s(d-2+\kappa)} \sum_{\nu}(2 \pi)^{d / 2}\left\|\sum_{j} \widehat{H_{j \nu}^{s}} \widehat{F_{j-s}}\right\|_{2}^{2} \\
& =C 2^{s(d-2+\kappa)} \sum_{\nu}\left\|\sum_{j} H_{j \nu}^{s} * F_{j-s}\right\|_{2}^{2} .
\end{aligned}
$$

For fixed $\nu$ write

$$
\begin{aligned}
\left\|\sum_{j=-\infty}^{\infty} H_{j \nu}^{s} * F_{j-s}\right\|_{2}^{2}= & \sum_{j=-\infty}^{\infty} \int \widetilde{H_{j \nu}^{s}} * H_{j \nu}^{s} * F_{j-s}(x) \overline{F_{j-s}}(x) d x \\
& +2 \sum_{j=-\infty}^{\infty} \sum_{i=-\infty}^{j-1} \int \widetilde{H_{j \nu}^{s}} * H_{i \nu}^{s} * F_{i-s}(x) \overline{F_{j-s}}(x) d x
\end{aligned}
$$

where $\widetilde{H_{j \nu}^{s}}(x)=\overline{H_{j \nu}^{s}}(-x)$.

Next observe that $\widetilde{H_{j \nu}^{s}} * H_{i \nu}^{s}$ is for $i \leq j$ supported in a rectangle $\mathcal{R}_{j \nu}^{s}$ centered at 0 with $d-1$ short sides of length $2^{j-s+10}$ and one long side of length $2^{j+10}$, the long side being parallel to $e_{\nu}^{s}$. Since the measure of $E_{\nu}^{s}$ is bounded by $C 2^{-s(d-1)}$ we have

$$
\left\|H_{i \nu}^{s}\right\|_{1} \lesssim \mathfrak{M}_{0} 2^{-s(d-1)}
$$

for all $i$ and consequently

$$
\left\|\widetilde{H_{j \nu}^{s}} * H_{i \nu}^{s}\right\|_{\infty} \leq\left\|H_{j \nu}^{s}\right\|_{\infty}\left\|H_{i \nu}^{s}\right\|_{1} \lesssim \mathfrak{M}_{0}^{2} 2^{-j d} 2^{-s(d-1)} .
$$

Therefore, since the cubes $Q$ are disjoint,

$$
\begin{aligned}
&\left|\widetilde{H_{j \nu}^{s}} * H_{j \nu}^{s} * F_{j-s}(x)\right|+2\left|\widetilde{H_{j \nu}^{s}} * \sum_{i<j} H_{i \nu}^{s} * F_{i-s}(x)\right| \\
& \lesssim\left[\mathfrak{M}_{0}\right]^{2} 2^{-j d} 2^{-s(d-1)} \int_{x+\mathcal{R}_{j \nu}^{s}} \sum_{i \leq j}\left|F_{i-s}(y)\right| d y \\
& \lesssim\left[\mathfrak{M}_{0}\right]^{2} 2^{-j d} 2^{-s(d-1)} \sum_{i} \sum_{\begin{array}{c}
L(Q)=i-s \\
Q \cap\left(x+\mathcal{R}_{j \nu}^{s}\right) \neq \emptyset
\end{array}} \int\left|f_{Q}(x)\right| d x \\
& \lesssim\left[\mathfrak{M}_{0}\right]^{2} 2^{-j d} 2^{-s(d-1)} \alpha \sum_{i} \sum_{\substack{L(Q)=i-s \\
Q \cap\left(x+\mathcal{R}_{j \nu}^{s}\right) \neq \emptyset}}|Q| \\
& \lesssim\left[\mathfrak{M}_{0}\right]^{2} 2^{-j d} 2^{-s(d-1)} \alpha\left|x+2 \mathcal{R}_{j \nu}^{s}\right| \\
& \lesssim\left[\mathfrak{M}_{0}\right]^{2} 2^{-s(2 d-2)} \alpha
\end{aligned}
$$


for all $x \in \mathbb{R}^{d}$. This finally implies that

$$
\begin{aligned}
\sum_{\nu}\left\|\sum_{j} H_{j \nu}^{s} * F_{j-s}\right\|_{2}^{2} & \lesssim\left[\mathfrak{M}_{0}\right]^{2} \alpha 2^{-s(2 d-2)} \operatorname{card}\left(\mathfrak{E}^{s}\right) \sum_{j}\left\|F_{j-s}\right\|_{1} \\
& \lesssim\left[\mathfrak{M}_{0}\right]^{2} \alpha 2^{-s(d-1)} \sum_{j}\left\|F_{j-s}\right\|_{1} \\
& \lesssim\left[\mathfrak{M}_{0}\right]^{2} \alpha 2^{-s(d-1)} \sum_{Q}\left\|f_{Q}\right\|_{1}
\end{aligned}
$$

and the asserted inequality follows from (2.4).

Proof of Lemma 2.2. Let $\beta \in C^{\infty}\left(\mathbb{R}^{d} \backslash\{0\}\right)$ be supported in $\{\xi: 1 / 2 \leq|\xi| \leq 2\}$ such that $\sum_{k} \beta^{2}\left(2^{-k} \xi\right)=1$ for all $\xi \neq 0$. Let $L_{k}$ be defined by $\widehat{L_{k}}(\xi)=\beta\left(2^{-k} \xi\right)$. Consider the multipliers

$$
m_{j \nu}^{s k}(\xi)=\beta\left(2^{-k} \xi\right)\left(1-\widehat{P_{\nu}^{s}}(\xi)\right) \widehat{H_{j \nu}^{s}}(\xi)
$$

then

$$
H_{j}-\Gamma_{j}^{s}=\sum_{\nu} \sum_{k} \mathcal{F}^{-1}\left[m_{j \nu}^{s k}\right] * L_{k} .
$$

Since $\operatorname{diam}(Q) \lesssim 2^{j-s}$ and $\left\|\nabla L_{k}\right\|_{1} \lesssim 2^{k}$ we obtain using the cancellation of $b_{Q}$

$$
\left\|\mathcal{F}^{-1}\left[m_{j \nu}^{s k}\right] * L_{k} * b_{Q}\right\|_{1} \lesssim\left\|\mathcal{F}^{-1}\left[m_{j \nu}^{s k}\right]\right\|_{1} \min \left\{1,2^{k+j-s}\right\}\left\|b_{Q}\right\|_{1} .
$$

Let $\ell_{s \nu}^{k}$ be the invertible linear transformation with $\ell_{s \nu}^{k} e_{\nu}^{s}=2^{k-s(1-\kappa)} e_{\nu}^{s}$ and $\ell_{s \nu}^{k} y=$ $2^{k} y$ if $\left\langle y, e_{\nu}^{s}\right\rangle=0$. It is straightforward to check that

$$
\left\|\partial_{\xi}^{\alpha}\left[\widehat{L_{k}}\left(1-\widehat{P_{\nu}^{s}}\right)\left(\ell_{s \nu}^{k} \cdot\right)\right]\right\|_{2} \leq C_{\alpha}
$$

for all multi-indices $\alpha$. Therefore $\widehat{L_{k}}\left(1-\widehat{P_{\nu}^{s}}\right)$ is an $L^{1}$ Fourier multiplier with norm independently of $k, s$ and $\nu$. Consequently

$$
\left\|\mathcal{F}^{-1}\left[m_{j \nu}^{s k}\right]\right\|_{1} \lesssim\left\|H_{j \nu}^{s}\right\|_{1} \lesssim 2^{-s(d-1)} \mathfrak{M}_{0} .
$$

In order to get a better bound for large $k$ we estimate $\widehat{H_{j \nu}^{s}}$ and its derivatives using integration by parts. Note that $1-\widehat{P_{\nu}^{s}}(\xi)=0$ if $\left|\left\langle\xi, e_{\nu}^{s}\right\rangle\right| \leq 2^{-s(1-\kappa)}|\xi|$. Therefore if $\theta \in E_{\nu}^{s}$ and if $\xi \in \operatorname{supp}\left(1-\widehat{P_{\nu}^{s}}\right)$ and $|\xi| \approx 2^{k}$, then $|\langle\theta, \xi\rangle| \gtrsim 2^{-s(1-\kappa)} 2^{k}$. Now

$$
\begin{aligned}
\widehat{H_{j \nu}^{s}}(\xi) & =\int \chi_{E_{\nu}^{s}}(\theta) \int H_{j}(r \theta) e^{-i r\langle\theta, \xi\rangle} r^{d-1} d r d \theta \\
& =\int \chi_{E_{\nu}^{s}}(\theta)(i\langle\theta, \xi\rangle)^{-N} \int \partial_{r}^{N} H_{j}(r \theta) e^{-i r\langle\theta, \xi\rangle} r^{d-1} d r d \theta
\end{aligned}
$$

Hence we obtain the size estimate

$$
\left|m_{j \nu}^{s k}(\xi)\right| \leq C_{N} \mathfrak{M}_{N}\left|E_{\nu}^{s}\right| 2^{[s(1-\kappa)-j-k)] N},
$$


uniformly in $\xi$. A similar calculation applies to the derivatives of $m_{j \nu}^{s k}$. Differentiating $\widehat{H_{j \nu}^{s}}$ yields additional factors of $r \approx 2^{j}$ in the above integral and differentiating $\beta\left(2^{-k} \cdot\right) \widehat{P_{\nu}^{s}}$ yields additional factors of $2^{s(1-\kappa)} 2^{-k}$. Since $\left|E_{\nu}^{s}\right| \leq C 2^{-s(d-1)}$ we see that

$$
\left\|\partial_{\xi}^{\alpha}\left[m_{j \nu}^{s k}\left(2^{k} \cdot\right)\right]\right\|_{2} \leq C_{\alpha} \mathfrak{M}_{N} 2^{-s(d-1)}\left(2^{s(1-\kappa)}+2^{j+k}\right)^{|\alpha|} 2^{-N(j+k-s(1-\kappa))}
$$

for all multi-indices $\alpha$ with $|\alpha| \leq N$. Therefore if $N \geq N_{1}>d / 2$

$$
\begin{aligned}
\left\|\mathcal{F}^{-1}\left[m_{j \nu}^{s k}\right]\right\|_{1} & \lesssim \sum_{|\alpha| \leq N_{1}}\left\|\partial_{\xi}^{\alpha}\left[m_{j \nu}^{s k}\left(2^{k} \cdot\right)\right]\right\|_{2} \\
& \lesssim \mathfrak{M}_{N} 2^{-s(d-1)} 2^{-N(j+k-s(1-\kappa))}\left(2^{(j+k) N_{1}}+2^{s(1-\kappa) N_{1}}\right) .
\end{aligned}
$$

Finally by $(2.5),(2.6)$

$$
\sum_{k \leq-j+s(1-\varepsilon)}\left\|\mathcal{F}^{-1}\left[m_{j \nu}^{s k}\right] * L_{k} * b_{Q}\right\|_{1} \lesssim \mathfrak{M}_{0} 2^{-s(d-1+\varepsilon)}\left\|b_{Q}\right\|_{1}
$$

and by (2.7) with $N_{1}=d, N \geq d+1$

$$
\begin{aligned}
& \sum_{k>-j+s(1-\varepsilon)}\left\|\mathcal{F}^{-1}\left[m_{j \nu}^{s k}\right] * L_{k} * b_{Q}\right\|_{1} \\
& \quad \lesssim \mathfrak{M}_{N} 2^{-s(d-1)} 2^{-s(\kappa-\varepsilon) N}\left[2^{s d(1-\varepsilon)}+2^{s d(1-\kappa)}\right]\left\|b_{Q}\right\|_{1}
\end{aligned}
$$

If we sum over $\nu$ and note that $\operatorname{card}\left(\mathfrak{E}^{s}\right) \lesssim 2^{s(d-1)}$, then (2.8) and (2.9) imply the statement of the lemma.

\section{Proof of the Theorem}

Clearly the $L^{p}$ boundedness for $1<p<\infty$ follows from the weak type $(1,1)$ estimate and the assumed $L^{2}$ boundedness, by a duality argument and the Marcinkiewicz interpolation theorem (see [7]). Therefore given $\lambda>0$ we have to verify the inequality

$$
\left|\left\{x \in \mathbb{R}^{d}:|T f(x)|>\lambda\right\}\right| \lesssim A \lambda^{-1}\|f\|_{1}
$$

where

$$
A=\|\widehat{K}\|_{\infty}+\int_{2}^{\infty} \eta(a) \frac{d a}{a}+\int_{S^{d-1}} V(\theta)\left(1+\log _{+}\left(V(\theta) /\|V\|_{1}\right)\right) d \theta ;
$$

here $\log _{+} s=\log s$ if $s \geq 1$ and $\log _{+} s=0$ where $0 \leq s<1$. Then by assumption $A<\infty$.

Given $f \in L^{1}\left(\mathbb{R}^{d}\right)$ we shall use the Calderón-Zygmund decomposition of $f$ at height $\alpha=\lambda / A$ (see Stein [7]). We decompose

$$
f=g+b=g+\sum_{Q} b_{Q}
$$


where $\|g\|_{\infty} \leq \alpha,\|g\|_{1} \lesssim\|f\|_{1}$, each $b_{Q}$ is supported in a dyadic cube $Q$ with sidelength $2^{L(Q)}$ and the cubes $Q$ have disjoint interiors. Moreover $\left\|b_{Q}\right\|_{1} \lesssim \alpha|Q|$ and $\sum_{Q}|Q| \lesssim \alpha^{-1}\|f\|_{1}$. For each $Q$ let $Q^{*}$ be the dilate of $Q$ with same center and $L\left(Q^{*}\right)=L(Q)+10$, and let $E=\bigcup Q^{*}$. Then also

$$
|E| \lesssim \alpha^{-1}\|f\|_{1}=A \lambda^{-1}\|f\|_{1} .
$$

Finally, for each $Q$, the mean value of $b_{Q}$ vanishes: $\int b_{Q}=0$. We shall use a variant of Calderón-Zygmund theory due to Fefferman [5] and modified by Christ [2].

As in standard Calderón-Zygmund theory we have the estimate for the good function $g$

$$
\|T g\|_{2}^{2} \leq\|T\|_{L^{2} \rightarrow L^{2}}^{2}\|g\|_{2}^{2} \leq A^{2}\|g\|_{1}\|g\|_{\infty} \leq A \lambda\|g\|_{1}
$$

and by Tshebyshev's inequality

$$
\left|\left\{x \in \mathbb{R}^{d}:|T g(x)|>\lambda / 2\right\}\right| \leq 4 \lambda^{-2}\|T g\|_{2}^{2} \leq 4 A \lambda^{-1}\|g\|_{1} \lesssim A \lambda^{-1}\|f\|_{1} .
$$

Therefore the proof of the Theorem is reduced to the estimate

$$
\left|\left\{x \in \mathbb{R}^{d} \backslash E:|T b(x)|>\lambda / 2\right\}\right| \lesssim A \lambda^{-1}\|f\|_{1} .
$$

Note that the expressions $T b_{Q}(x)$ are well defined for almost all $x \in \mathbb{R}^{d} \backslash E$ since we assume that $K$ is locally integrable away from the origin.

We now introduce a dyadic decomposition of the kernel. Let $\beta \in C_{0}^{\infty}\left(\mathbb{R}_{+}\right)$be as in the previous section $\left(\operatorname{supp} \beta \subset(1 / 2,2), \sum_{k} \beta^{2}\left(2^{-k} t\right)=1\right.$ for all $\left.t>0\right)$. Define

$$
K_{j}(x)=\beta^{2}\left(2^{-j}|x|\right) K(x) .
$$

For $m \in \mathbb{Z}$ let

$$
B_{m}=\sum_{L(Q)=m} b_{Q}
$$

Then observe that the support of the functions $K_{j} * B_{j-s}$ is contained in $E$ if $s \leq 3$. Therefore, in order to verify (3.2), it suffices to prove that

$$
\left|\left\{x \in \mathbb{R}^{d}:\left|\sum_{s>3} \sum_{j} K_{j} * B_{j-s}(x)\right|>\lambda / 2\right\}\right| \lesssim A \lambda^{-1}\|f\|_{1} .
$$

We now decompose the kernels $K_{j}$ in the spherical variables according to the size of $V$; moreover, we introduce a regularization in the radial variable.

Let

$$
\delta=[100(d+2)]^{-1}
$$

and let

$$
D^{s}=\left\{\theta \in S^{d-1}: V(\theta) \leq 2^{\delta s}\|V\|_{L^{1}\left(S^{d-1}\right)}\right\} .
$$

Let $\phi \in C_{0}^{\infty}(\mathbb{R})$ such that $\int \phi(t) d t=1$ and such that $\phi(t)=0$ if $|t| \geq 2^{-10}$. Then

$$
K_{j}=H_{j}^{s}+R_{j}^{s}+S_{j}^{s}
$$


where

$$
\begin{aligned}
S_{j}^{s}(r \theta) & =\beta^{2}\left(2^{-j} r\right)\left[K(r \theta)-\int K(\rho \theta) 2^{\delta s-j} \phi\left(2^{\delta s-j}(r-\rho)\right) d \rho\right], \\
R_{j}^{s}(r \theta) & =\beta^{2}\left(2^{-j} r\right) \chi_{S^{d-1} \backslash D^{s}}(\theta) \int K(\rho \theta) 2^{\delta s-j} \phi\left(2^{\delta s-j}(r-\rho)\right) d \rho, \\
H_{j}^{s}(r, \theta) & =\beta^{2}\left(2^{-j} r\right) \chi_{D^{s}}(\theta) \int K(\rho \theta) 2^{\delta s-j} \phi\left(2^{\delta s-j}(r-\rho)\right) d \rho .
\end{aligned}
$$

Observe that $H_{j}^{s}$ vanishes if $|x| \notin\left[2^{j-2}, 2^{j+2}\right]$ and that for $2^{j-2} \leq r \leq 2^{j+2}, \theta \in D^{s}$,

$$
r^{d+l}\left|\left(\frac{\partial}{\partial r}\right)^{l} H_{j}^{s}(r, \theta)\right| \leq C_{l} 2^{\delta s(l+1)} \int_{r / 2}^{2 r}|K(\rho \theta)| \rho^{d-1} d \rho \leq 2 C_{l} 2^{\delta s(l+2)}\|V\|_{L^{1}\left(S^{d-1}\right)} .
$$

That is, for fixed $s>3$ and for all $N$, the family $\left\{H_{j}^{s}\right\}$ satisfies the assumption (2.1) with

$$
\mathfrak{M}_{N}=C_{N}\|V\|_{L^{1}\left(S^{d-1}\right)} 2^{\delta s(N+2)}
$$

where $C_{N}$ does not depend on $V$ or $s$. We now decompose

$$
H_{j}^{s}=\Gamma_{j}^{s}+\left(H_{j}^{s}-\Gamma_{j}^{s}\right)
$$

exactly as in (2.2), except that this time the operator $H_{j}$ itself depends on $s$. The decomposition (2.2) depended on a parameter $0<\kappa<1$; we may now choose $\kappa=1 / 2$.

We have split

$$
\sum_{s>3} \sum_{j} K_{j} * B_{j-s}(x)=I(x)+I I(x)
$$

where

$$
I(x)=\sum_{s>3} \sum_{j} \Gamma_{j}^{s} * B_{j-s}(x)
$$

and

$$
I I(x)=\sum_{s>3} \sum_{j}\left[H_{j}^{s}-\Gamma_{j}^{s}+R_{j}^{s}+S_{j}^{s}\right] * B_{j-s}(x) .
$$

Now by Tshebyshev's inequality

$$
\begin{aligned}
\mid\{x \in & \left.\mathbb{R}^{d}:\left|\sum_{s>3} \sum_{j} K_{j} * B_{j-s}(x)\right|>\lambda / 2\right\} \mid \\
& \leq\left|\left\{x \in \mathbb{R}^{d}:|I(x)|>\lambda / 4\right\}\right|+\left|\left\{x \in \mathbb{R}^{d}:|I I(x)|>\lambda / 4\right\}\right| \\
& \leq 16 \lambda^{-2}\|I\|_{2}^{2}+4 \lambda^{-1}\|I I\|_{1} .
\end{aligned}
$$

By Lemma 2.1 and (3.5) with $N=0$ we have

$$
\begin{aligned}
\|I\|_{2}^{2} & \leq\left[\sum_{s>3}\left\|\sum_{j} \Gamma_{j}^{s} * B_{j-s}\right\|_{2}\right]^{2} \\
& \lesssim\|V\|_{L^{1}\left(S^{d-1}\right)}^{2}\left[\sum_{s>3}\left(2^{s(4 \delta+\kappa-1)} \alpha \sum_{Q}\left\|b_{Q}\right\|_{1}\right)^{1 / 2}\right]^{2} \\
& \lesssim A^{2} \alpha \sum_{Q}\left\|b_{Q}\right\|_{1} \lesssim A \lambda\|f\|_{1}
\end{aligned}
$$


here we could sum the geometrical series since $4 \delta+\kappa-1<-1 / 4$.

Next we apply Lemma 2.2 with $N=5(d+1), \varepsilon=1 / 4$ and obtain

$$
\begin{aligned}
\left\|\sum_{s>3} \sum_{j}\left(H^{s}-\Gamma_{j}^{s}\right) * B_{j-s}\right\|_{1} & \lesssim \sum_{s>3} A 2^{(N+2) \delta s}\left(2^{-\varepsilon s}+2^{s(d-(\kappa-\varepsilon) N)}\right) \sum_{j}\left\|B_{j-s}\right\|_{1} \\
& \lesssim A\|f\|_{1}
\end{aligned}
$$

now we have used that $(N+2) \delta+d-(\kappa-\varepsilon) N<-(d+1) / 4$ and $(N+2) \delta-\varepsilon<-1 / 8$.

It remains to estimate the sums involving $R_{j}^{s}$ and $S_{j}^{s}$. Observe that

$$
\left\|R_{j}^{s}\right\|_{1} \leq \int_{S^{d-1} \backslash D^{s}} \int_{2^{j-1}}^{2^{j+1}}|K(r \theta)| r^{d-1} d r d \theta \lesssim \int_{V(\theta)>2^{\delta s}\|V\|_{1}} V(\theta) d \theta
$$

and therefore

$$
\begin{aligned}
\| \sum_{s>3} & \sum_{j} R_{j}^{s} * B_{j-s}\left\|_{1} \lesssim \sum_{s>3} \sum_{j}\right\| B_{j-s} \|_{1} \int_{V(\theta)>2^{\delta s}\|V\|_{1}} V(\theta) d \theta \\
& \lesssim \sum_{Q}\left\|b_{Q}\right\|_{1} \int V(\theta) \operatorname{card}\left(\left\{s \in \mathbb{N}: 2^{\delta s} \leq|V(\theta)| /\|V\|_{1}\right\}\right) d \theta \\
& \lesssim \int V(\theta)\left(1+\log _{+}\left(V(\theta) /\|V\|_{1}\right)\right) d \theta \sum_{Q}\left\|b_{Q}\right\|_{1} \\
& \lesssim A\|f\|_{1} .
\end{aligned}
$$

Finally $\left\|S_{j}^{s}\right\|_{1} \lesssim \sup _{R}\left\|V_{R}\right\|_{1}$ and for $s>10 / \delta$

$$
\begin{aligned}
\left\|S_{j}^{s}\right\|_{1} & \leq \iiint_{2^{j-1}}^{2^{j+1}}|K((r-\rho) \theta)-K(r \theta)| r^{d-1} d r d \theta\left|2^{\delta s-j} \phi\left(2^{\delta s-j} \rho\right)\right| d \rho \\
& \lesssim \eta\left(2^{\delta s-3}\right) .
\end{aligned}
$$

Therefore

$$
\begin{aligned}
& \left\|\sum_{s>3} \sum_{j} S_{j}^{s} * B_{j-s}\right\|_{1} \\
& \lesssim \sum_{0<s<10 / \delta} \sum_{j}\left\|B_{j-s}\right\|_{1} \sup _{R}\left\|V_{R}\right\|_{L^{1}\left(S^{d-1}\right)} \\
& \quad+\sum_{s>10 / \delta} \sum_{j}\left\|B_{j-s}\right\|_{1} \eta\left(2^{\delta s-3}\right) \\
& \lesssim\left[\sup _{R}\left\|V_{R}\right\|_{L^{1}\left(S^{d-1}\right)}+\int_{2}^{\infty} \eta(a) \frac{d a}{a}\right] \sum_{Q}\left\|b_{Q}\right\|_{1} \\
& \lesssim A\|f\|_{1} .
\end{aligned}
$$

Now by (3.8), (3.9) and (3.10)

$$
\|I I\|_{1} \lesssim A\|f\|_{1}
$$


and the desired weak type inequality (3.3) follows from equations (3.6), (3.7) and (3.11).

We conclude by proving the remark following the statement of the Theorem. We have to change the definitions of the functions $H_{j}^{s}$ and $R_{j}^{s}$ in (3.4). Let $C=$ $\sup _{R}\left\|V_{R}\right\|_{1}$ and for $j \in \mathbb{Z}$

$$
D_{j}^{s}=\left\{\theta \in S^{d-1}: V_{2^{j-1}}(\theta)+V_{2^{j}}(\theta)+V_{2^{j+1}}(\theta) \leq 2^{2+\delta s} C\right\} .
$$

In the present setting we define $H_{j}^{s}$ and $R_{j}^{s}$ as before but with $D^{s}$ replaced by $D_{j}^{s}$. The estimate (3.9) is changed to

$$
\begin{aligned}
& \left\|\sum_{s>3} \sum_{j} R_{j}^{s} * B_{j-s}\right\|_{1} \\
& \quad \lesssim \sum_{s>3} \sum_{j}\left\|B_{j-s}\right\|_{1} \sum_{\sigma=-1}^{1} \int_{V_{2^{j+\sigma}}(\theta)>2^{\delta s} C} V_{2^{j+\sigma}}(\theta) d \theta \\
& \quad \lesssim\|f\|_{1} \sum_{s>3} \sup _{j} \sum_{\sigma=-1}^{1} \int_{V_{2^{j+\sigma}}(\theta)>2^{\delta s} C} V_{2^{j+\sigma}}(\theta) d \theta \\
& \quad \lesssim\|f\|_{1} \sum_{s>3} \frac{1}{\Delta\left(2^{\delta s} / C\right)} \sup _{j} \sup _{s} \sum_{\sigma=-1}^{1} \int V_{2^{j+\sigma}}(\theta) \Delta\left(V_{2^{j+\sigma}}(\theta) / C\right) d \theta \\
& \quad \lesssim\|f\|_{1} \int_{1}^{\infty} \frac{d a}{a \Delta(a)} \sup _{R} \int V_{R}(\theta) \Delta\left(V_{R}(\theta) / C\right) d \theta .
\end{aligned}
$$

The other estimates remain essentially unchanged; in various instances one replaces $\|V\|_{1}$ by $\sup _{R}\left\|V_{R}\right\|_{1}$.

\section{REFERENCES}

1. A. P. Calderón and A. Zygmund, On singular integrals, Amer. J. Math. 78 (1956), 289-309. MR 18:894a

2. M. Christ, Weak type $(1,1)$ bounds for rough operators, Ann. of Math. (2) 128 (1988), 19-42. MR 89m: 42013

3. M. Christ and J.-L. Rubio de Francia, Weak type $(1,1)$ bounds for rough operators, II, Invent. Math. 93 (1988), 225-237. MR 90d:42021

4. M. Christ and C. D. Sogge, The weak type $L^{1}$ convergence of eigenfunction expansions for pseudo-differential operators, Invent. Math. 94 (1988), 421-453. MR 89j:35096

5. C. Fefferman, Inequalities for strongly singular convolution operators, Acta Math. 124 (1970), 9-36. MR 41:2468

6. S. Hofmann, Weak $(1,1)$ boundedness of singular integrals with nonsmooth kernel, Proc. Amer. Math. Soc. 103 (1988), 260-264. MR 89f:42013

7. E. M. Stein, Singular integrals and differentiability properties of functions, Princeton Univ. Press, Princeton, NJ, 1971. MR 44:7280

Department of Mathematics, University of Wisconsin, Madison, Wisconsin 53706

E-mail address: seeger@math.wisc.edu 